\title{
Solvent-Assisted Surface Engineering for High-Performance All- Inorganic Perovskite Nanocrystal Light-Emitting Diodes
}

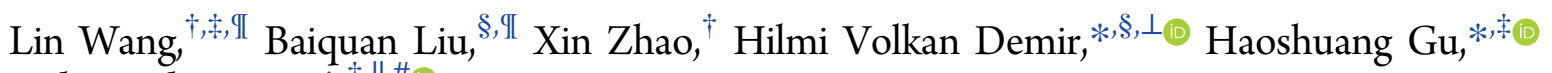 \\ and Handong Sun ${ }^{*},, \|, \# \mathbb{C}$
}

\begin{abstract}
${ }^{\dagger}$ Division of Physics and Applied Physics, School of Physical and Mathematical Sciences, Nanyang Technological University, Singapore 637371, Singapore

${ }^{\ddagger}$ Hubei Collaborative Innovation Center for Advanced Organic Chemical Materials, Hubei Key Laboratory of Ferro \& Piezoelectric Materials and Devices, Faculty of Physics \& Electronic Sciences, Hubei University, Wuhan 430062, China

${ }^{\S}$ Luminous! Center of Excellence for Semiconductor Lighting and Displays, School of Electrical and Electronic Engineering and School of Physical and Mathematical Sciences, Nanyang Technological University, Singapore 639798, Singapore

"Centre for Disruptive Photonic Technologies (CDPT), School of Physical and Mathematical Sciences, Nanyang Technological University, 21 Nanyang Link, 637371, Singapore

${ }^{\perp}$ Department of Electrical and Electronics Engineering, Department of Physics, UNAM-Institute of Materials Science and Nanotechnology, Bilkent University, Ankara 06800, Turkey

${ }^{\#}$ MajuLab, CNRS-UCA-SU-NUS-NTU International Joint Research Unit, Singapore
\end{abstract}

\section{Supporting Information}

\begin{abstract}
All-inorganic cesium halide perovskite nanocrystals have attracted much interest in optoelectronic applications for the sake of the readily adjustable band gaps, high photoluminescence quantum yield, pure color emission, and affordable cost. However, because of the ineluctable utilization of organic surfactants during the synthesis, the structural and optical properties of $\mathrm{CsPbBr}_{3}$ nanocrystals degrade upon transforming from colloidal solutions to solid thin films, which plagues the device operation. Here, we develop a novel solvent-assisted surface engineering strategy, producing high-quality $\mathrm{Cs} \mathrm{PbBr}_{3}$ thin films for device applications. A good solvent is first introduced as an assembly trigger to conduct assembly in a one-dimensional direction, which is then interrupted by adding a nonsolvent. The nonsolvent drives the adjacent nanoparticles connecting in a two-dimensional direction. Assembled $\mathrm{CsPbBr}_{3}$ nanocrystal thin films are densely packed and very smooth with a surface roughness of $\sim 4.8 \mathrm{~nm}$, which is highly desirable for carrier transport in a light-emitting diode (LED) device. Meanwhile, the film stability is apparently improved. Benefiting from this facile and reliable strategy, we have achieved remarkably improved performance of $\mathrm{CsPbBr}_{3}$ nanocrystal-based LEDs. Our results not only enrich the methods of nanocrystal surface engineering but also shed light on developing high-performance LEDs.
\end{abstract}

KEYWORDS: $\mathrm{CsPbBr}$ nanocrystals, solvent engineering, self-assembly, photoluminescence, light-emitting diodes

\section{INTRODUCTION}

The all-inorganic cesium lead halide perovskites (ILHPs), as the name implies, have a crystal structure similar to oxide perovskites, which contains a cubic lattice with cornerconnected $\left[\mathrm{PbX}_{6}\right]$ octahedras in three dimensions and yields a general $\mathrm{APbX}_{3}(\mathrm{~A}=\mathrm{Cs}, \mathrm{X}=\mathrm{Cl}, \mathrm{Br}, \mathrm{I})$ composition. Since the report by Kovalenko's group in 2015, ${ }^{1}$ ILHPs nanocrystals have attracted much attention because of not only their excellent optical performances, that is, bright and adjustable photoluminescence $(\mathrm{PL})$ controlled by halide anions or particle size, but also because of their cost-effective fabrication approaches such as the high-regulation hot injection synthesis, the facile room-temperature synthesis, and the fast anion exchange methods. $^{2-6}$ So far, a bunch of different nanostructures of the ILHPs including nanocubes, ${ }^{7}$ nanorods, ${ }^{8}$ nanowires, ${ }^{9}$ nanoplatelets, ${ }^{10}$ nanosheets ${ }^{11}$ have been extensively studied and reported. Especially, the utilization of ILHP nanocrystals has led to the development of promising optoelectronic devices, ${ }^{12}$ such as light-emitting diodes (LEDs), ${ }^{13-16}$ photodetectors, ${ }^{2,17,18}$ solar cells, ${ }^{19,20}$ and lasers. ${ }^{21-23}$ It is well-known that the performance of an electrically driven device depends on the properties of both the active material in the form of the thin film and the carrier injection scheme [electron and hole transport layers (HTLs)]. Regarding ILHP nanocrystal-based devices (e.g., LEDs), most of the attention has been paid on engineering the carrier injection layers to improve the carrier

Received: April 16, 2018

Accepted: May 18, 2018

Published: May 18, 2018 
injection efficiency and/or transport. Efforts of optimizing active materials have been mainly focused on improving the quantum yields of nanocrystals in solution rather than on how to improve the quality of nanocrystal thin films. However, the structure and performance integrity of the $\mathrm{CsPbX}_{3}(\mathrm{X}=\mathrm{Cl}, \mathrm{Br}$, I) nanocrystals degrade upon transforming to the form of thin films. Improperly processed nanocrystals will bring about nanocrystal clustering and form a very rough thin film, which deteriorates not only the charge transport and emission efficiency but also the device stability. The surface ligands, owing to the poor electric conductivity, will inevitably build an insulating barrier when the nanocrystals are assembled into thin films. ${ }^{24}$ The barrier layer of ligands may block the charges injection into the perovskite emission layer, resulting in low carrier injection efficiency, poor charge transport balance, and inferior external quantum efficiency (EQE). ${ }^{25}$ On the other side, the retention of long-term structural integrity of the colloidal nanocrystals counts on the coverage of ligand chains, which help to retain high PL quantum yield (PLQY) and good dispersion in solution. ${ }^{26}$ Thus, the overall performance is a matter of trade-off between the PLQY and the charge transport. Unlike the conventional semiconductor quantum dots (QDs), the unique merit of high defect tolerance of $\mathrm{CsPbBr}_{3}$ nanocrystals endows them with strong immunity to the electronic quality degradation under the existence of carriertrapping centers, such as abundant antisite and interstitial point defects. $^{27,28}$

To obtain high-quality ILHPs thin films from nanocrystal solution, a plausible strategy is the healing treatment of the asfabricated solid films, ${ }^{29,30}$ which is a holistic passivation process but easily results in uncontrollable cracks in the whole layer because of the volume contraction and unexpected redissolution of ILHPs. The alternative strategy is, first, to preameliorate the colloidal ILHPs nanocrystals and then fabricate the thin film, ${ }^{13,31-34}$ which is obviously more efficient in regulating the thin film. Thanks to the sensitivity of ILHPs to solvent polarity and the dynamic ligand binding modes, ${ }^{26}$ the protogenetic surface ligands could be mediated through the surface engineering, which incidentally gives an opportunity to the demanded assembly of ILHPs via the effect of the solvent polarity. ${ }^{35}$ The controlled assembly of the ILHPs nanocrystals is favorable to the simultaneous retainment of reasonably high PLQY and structure integrity of the ILHP crystal structure and thus to the formation of high-quality thin films. ${ }^{36}$

In this work, we proposed a facile strategy, solvent-assisted surface engineering, to allow ILHP nanocrystals to retain the structural integrity and reasonably high PLQY as well as to enable densely packed smooth thin films. The high-quality $\mathrm{CsPbBr}$ thin films with enhanced photoirradiation stability and much better transport performance were achieved with the aid of the nanocrystal self-assembly behavior during solvent evaporation. We validate our strategy by demonstrating highly efficient $\mathrm{CsPbBr}_{3}$ green LEDs with the maximum EQE of $1.70 \%$, which is 34 -fold enhancement than the control device based on unprocessed $\mathrm{CsPbr}_{3}$ nanocrystals. The maximum power efficiency is 40 -fold improvement, and the full width at half maximum (fwhm) of the electroluminescence (EL) spectrum is as narrow as $16 \mathrm{~nm}$.

\section{RESULTS AND DISCUSSION}

It is well-known that self-assembly or self-organization of colloidal nanocrystals, acting as the artificial blocks, into 1-, 2or 3-dimensional structures is an initiative process actuated by the interactions inside the nanocrystal solution such as entropy, electrostatic force, van der Waals force, and hydrogen bonding forces. ${ }^{37}$ The dominated interaction depends on the competition between the repulsive and attractive forces, which could be controlled by the chain length of ligands, the size and shape of the nanocrystals, and the solvent polarity. ${ }^{38}$ In this work, the $\mathrm{CsPbBr}{ }_{3}$ nanocrystals were synthesized by a modified hotinjection method using oleic acid (OA) and oleylamine (OLA) as surfactants, ${ }^{1,7}$ presenting a cubic shape with a mean size of $\sim 10 \mathrm{~nm}$ (Figure S1, Supporting Information). The surfactant molecules are adsorbed on nanocrystal surfaces via covalent bonds (Figure S2, Supporting Information), forming an organic-inorganic interface which protects the nanocrystal surfaces and facilitates to disperse in solution. Because of the surface-capping ligand chains, there exists a ligand corona around the planar crystalline surfaces, so that the real shape of the nanocrystals when dispersed in solvent is like quasispherical, ${ }^{39}$ and the radius $(r)$ should be $a / 2+l,(a$ is the side length of cubic core, $l$ is the length of the ligand chain $\sim 2 \mathrm{~nm}$ ), as shown in Figure 1a. It implies that besides the core-core

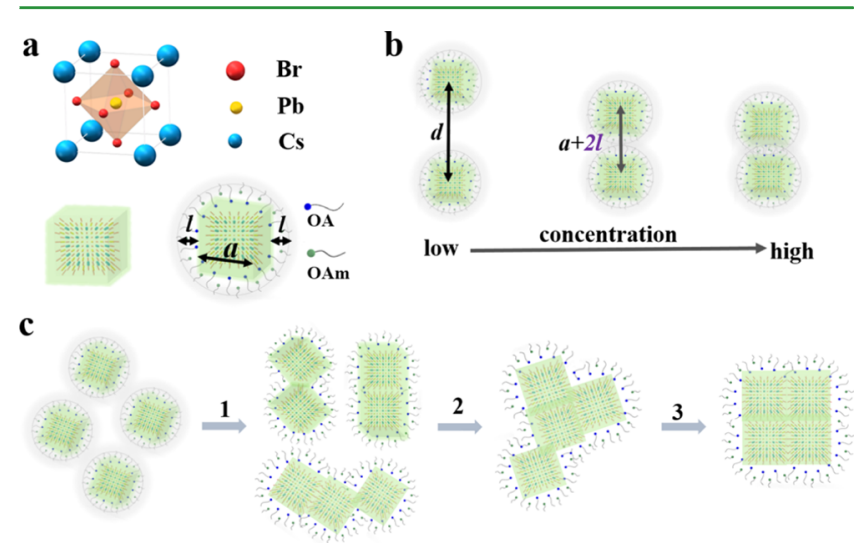

Figure 1. Schematic illustration of (a) crystal structure, the ligands capped nanocrystal and (b) interaction between nanoparticles change with the concentration. (c) Illustration of the model for solventassisted interface engineering: step 1 is the solvent trigger process to obtain one-dimensional assembles, step 2 is to terminate the former process and obtain two-dimensional assemblies, and step 3 represents the thin film assembly during solvent evaporation. The corresponding experiment procedures are depicted in Figure S3 (Supporting Information).

interaction, both the corona-corona and ligand-core interactions affect the assembly behavior of the $\mathrm{CsPbBr}_{3}$ nanocrystals upon solvent evaporation. The corona morphology would change with the variation of the nanocrystal concentration as well as the switch of the interparticle interactions. ${ }^{37}$ As exhibited in Figure 1b, the interparticle distance $(d)$ decreases with the crowding of the nanoparticles in a certain volume of solvents. At low concentrations, $d$ is larger than $a+2 l$, when the repulsive potential dominates and no flocculation occurs. The repulsion force is favorable to the monodispersing of the nanoparticles. The nanoparticles suffer from fierce competition between corona-corona repulsion and core-core attraction forces when $d$ equals to $a+2 l$. With the increase of the concentration, the coronas would first experience the selfcompression to avoid the aggregation and then the interpenetration due to the domination of the attraction force. This is coincident with the general self-aggregation of nanoparticles during the solvent evaporation process. ${ }^{24,26,28,40,41}$ 

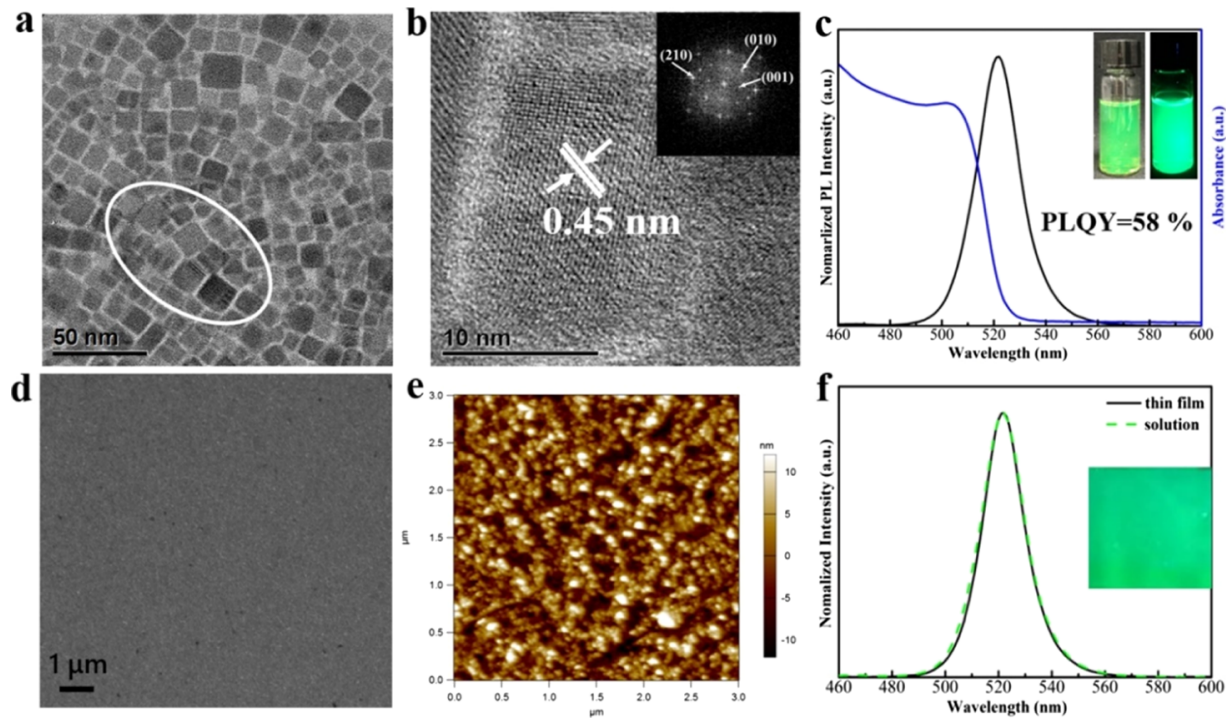

Figure 2. Effect of the solvent-assisted interface engineering on $\mathrm{CsPbr}_{3}$ nanocrystal performances. (a) Low magnification and (b) HRTEM images of the assembled $\mathrm{CsPbBr}_{3}$ nanocrystals; inset is the corresponding fast Fourier transform image. (c) UV-vis absorbance and $\mathrm{PL}$ spectra of $\mathrm{CsPbBr}$ nanocrystals dispersed in hexane and photographs under daylight (left inset) and $365 \mathrm{~nm}$ UV light (right inset). (d) SEM and (e) AFM images of the spin-coating-casted $\mathrm{CsPbBr}_{3}$ thin film. (f) $\mathrm{PL}$ spectrum (black solid line) and photograph (under $365 \mathrm{~nm} \mathrm{UV}$ light) of the CsPbBr ${ }_{3}$ thin film. The PL spectrum in the green dashed line represents $\mathrm{CsPbBr}_{3}$ nanocrystals in hexane.

As is shown in Figure 1c, taking advantage of the dynamic ligand-surface interface and the sensitivity of ILHPs to solvent polarity, the good solvent was utilized as the assembly trigger (step 1) to conduct the assemble process in a one-dimensional orientation because of the collaboration effect of the hydrophobic effect and van der Waals interaction. ${ }^{35}$ Under high density condition, the interparticle distance will get smaller, and the nanoparticles have the potential to orderly assemble to maximize the system's total entropy. ${ }^{37} \mathrm{~A}$ nonsolvent or antisolvent was then employed (step 2) to terminate the onedimensional assembling process and drive the organization in two-dimensional orientation, by virtue of the maximum of the interparticle attractive forces. ${ }^{35}$ The solution destabilization makes ligand coronas contract together to screen the poor solvent permeation, and some ligands would desorb from the surface to minimize the corona surface area. In view of the fragile nature of $\mathrm{CsPBr}_{3}$, the hexane (polarity index is 0.06) and ethyl acetate (EA, polarity index is 4.3 ) were chosen as the good solvent and the nonsolvent, respectively. ${ }^{36,42}$ The subsequent discard of the nonsolvent ends the assembly process in the two-dimensional direction and makes the final products possess a finite assembled size as well as the good dispersity and stability in solution, which is beneficial to the thin film fabrication during the drying of solvents (step 3). Therefore, the solvent-assisted surface engineering, activating the nanoparticle self-assembly behaviors, ensures the fabrication of dense and smooth $\mathrm{CsPBB}_{3}$ nanoparticle thin films. The corresponding experiment details are depicted and described in Figure S3 (Supporting Information).

Thanks to the ionic nature of the $\mathrm{Cs} \mathrm{PbBr}_{3}$ crystal structure, the crowding nanoparticles could continue to grow and gather together to rearrange their overall appearance. As shown in Figure 2a, the as-synthesized $\mathrm{CsPbBr}_{3}$ nanocrystals exhibit nanocube morphology, and it seems like, from the whole view, the assembly of the nanocubes into a one-dimensional structure has been first interrupted by growing in planar two-dimensional direction, which is also frozen at the intermediate state resulting in some bigger nanocubes with a size of $\sim 25 \mathrm{~nm}$. This phenomenon is very consistent with the expected results from solvent-assisted surface engineering. When treated with the typical antisolvents (e.g., acetone) purification processes, the $\mathrm{CsPbBr}{ }_{3}$ nanoparticles appear to be dispersed in isolation as shown in Figure S1 (Supporting Information). ${ }^{1,7,36} \mathrm{CsPbBr}_{3}$ nanoparticles processed by the proposed solvent-assisted surface engineering presents distinctly localized assembly in both one-dimensional and two-dimensional directions. For example, as shown in the white circle area in Figure 2a, the adjacent nanoparticles are connected by the means of face-toface, edge-to-edge, or corner-to-corner contacts.

To get deep insight into the way how the separated nanocubes fuse together, the high-resolution transmission electron microscopy (HRTEM) image in Figure $2 \mathrm{~b}$ reveals that two independent nanocubes integrate directly, and the well-resolved interfringe distance of $0.45 \mathrm{~nm}$ at the combining interface could be indexed to the (010) lattice spacings of the cubic phase (Figure $2 \mathrm{~b}$ inset), and the assembly process could also be conducted along the -202 direction (Figure S4, Supporting Information). The optical properties could also demonstrate the interconnecting between nanocubes. As shown in Figure 2c, the PL peak of $522 \mathrm{~nm}$ is slightly red-shifted from that of pristine $\mathrm{CsPbBr}$ nanoparticles with a PL peak at 520 $\mathrm{nm}$ (Figure S5, Supporting Information), which indirectly verifies the size growth of some nanoparticles after the solventassisted surface engineering treatment. ${ }^{7}$ In addition, the decrease of the PLQY from $90 \%$ to $58 \%$ indicates that the $\mathrm{CsPBBr}_{3}$ nanocubes were assembled at the expense of the surface ligands. ${ }^{36}$ Meanwhile, the resultant assembled $\mathrm{CsPbBr}_{3}$ nanoparticles still retain good dispersity in the solution (insets in Figure 2c). Remarkably, all these results are in good agreement with what is expected from the proposed assembly mechanism. The $\mathrm{CsPbBr}_{3}$ thin film was fabricated by spincoating technique. The scanning electron microscopy (SEM) and atomic force microscopy (AFM) images (Figure 2d,e) demonstrate that the thin film was highly dense and very smooth with a surface roughness of $4.8 \mathrm{~nm}$. Comparatively, the thin film made from unassembled $\mathrm{CsPbBr}_{3}$ nanocrystals 

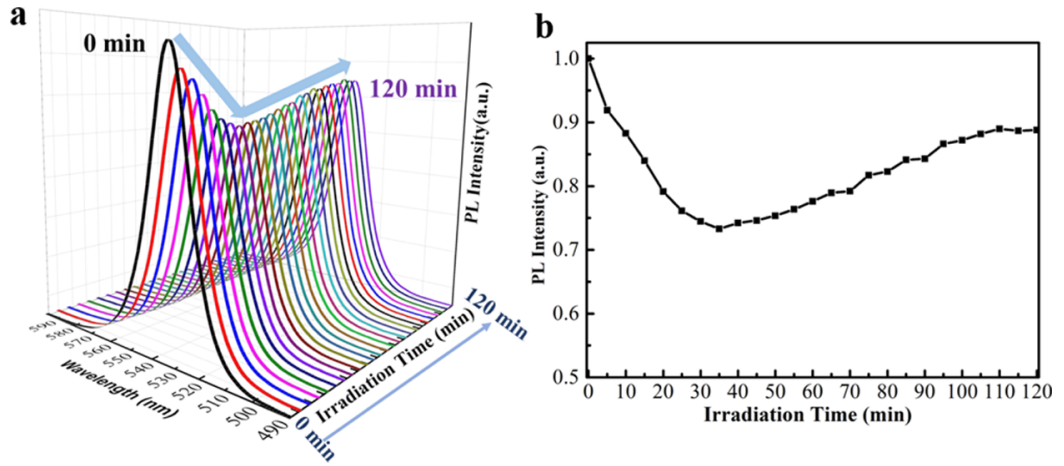

Figure 3. Photostability performance of the $\mathrm{CsPbBr}_{3}$ thin film. (a) In situ PL spectra were collected every 5 min during the continuous irradiation (excitation wavelength of $442 \mathrm{~nm}$ and intensity of $50 \mathrm{~mW} \mathrm{~cm}^{-2}$ ). (b) PL intensity versus the light exposure time.

a

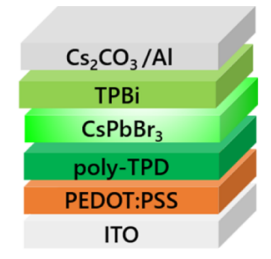

c

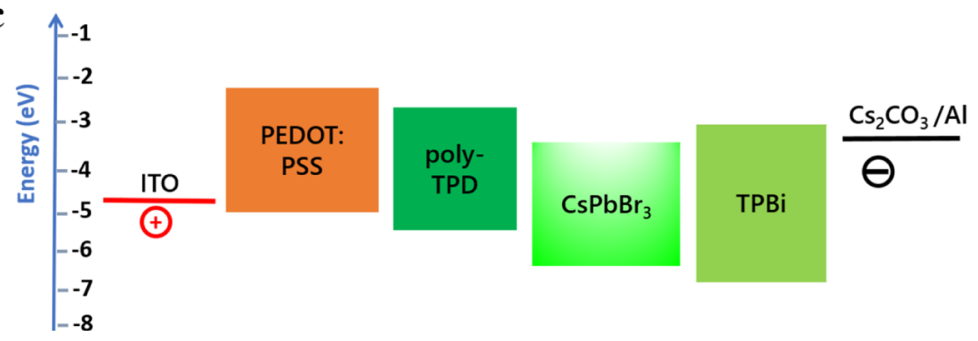

b
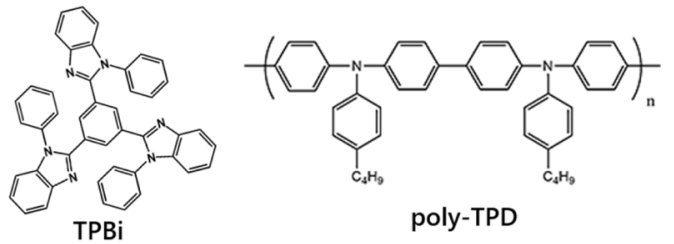

poly-TPD 

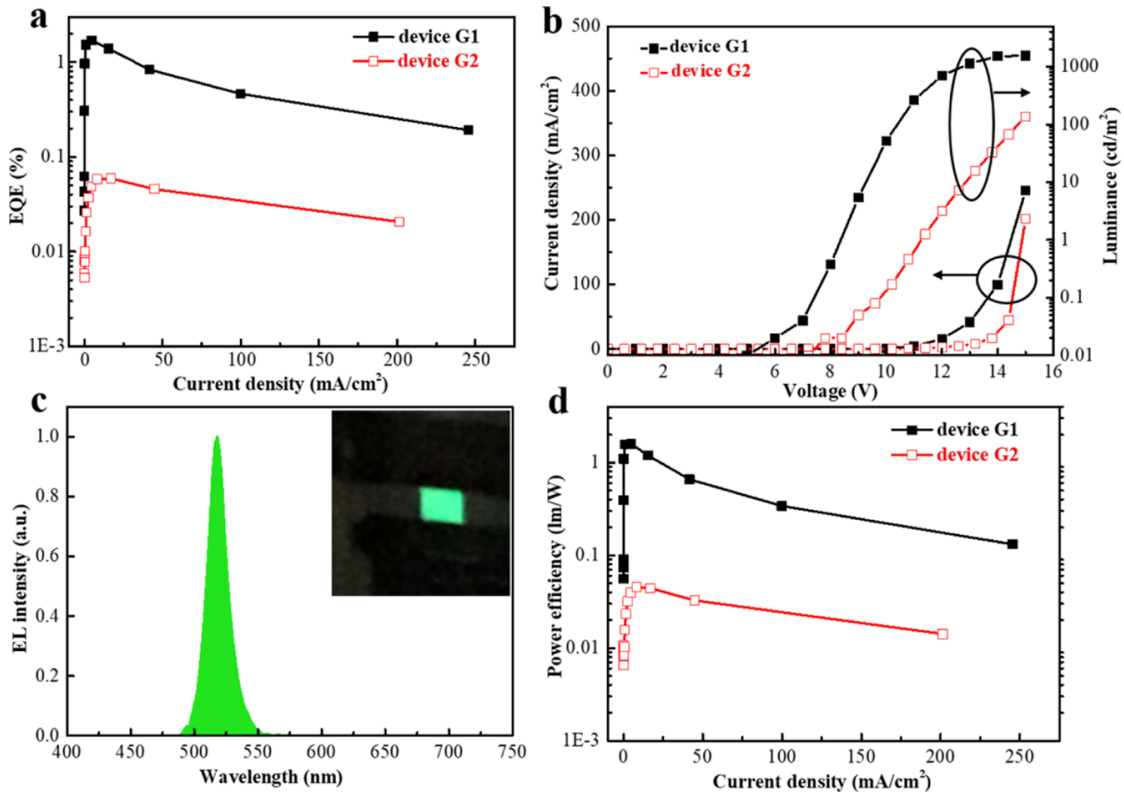

Figure 5. Performances of the device G1 and the control device G2. (a) EQE as a function of current density. (b) Current density and luminance versus driving voltage. (c) Normalized EL spectrum at an applied voltage of $10 \mathrm{~V}$ and a photograph of device G1 under bias (inset). (d) Power efficiency as a function of current density.

developed. The device configuration is shown in Figure 4a, composed of multilayers in the following order: indium tin oxide (ITO), poly(ethylenedioxythiophene):polystyrene sulfonate (PEDOT:PSS, $40 \mathrm{~nm}), N, N^{\prime}$-bis(4-butylphenyl)- $N, N^{\prime}$ bis(phenyl)benzidine (poly-TPD, $40 \mathrm{~nm}$ ), CsPbBr $\mathrm{CPmitting}_{3}$ layer $(20 \mathrm{~nm}), 2,2^{\prime}, 2^{\prime \prime}$-(1,3,5-benzenetriyl)tris-1-phenyl-1Hbenzimidazole (TPBi, $35 \mathrm{~nm})$, and $\mathrm{Cs}_{2} \mathrm{CO}_{3} / \mathrm{Al}(1 / 100 \mathrm{~nm})$. Except for the TPBi and $\mathrm{LiF} / \mathrm{Al}$ layers which were deposited with thermal vacuum technology, all the other layers were fabricated by spin coating layer by layer on a prepatterned ITO transparent anode. The $\mathrm{CsPbBr}$-emitting layer built on the HTL layer exhibited homogenous density and smooth morphology, with a surface roughness of $4.75 \mathrm{~nm}$ (Figures S13 and S14, Supporting Information). The cross-sectional SEM image of the PeLED is depicted in Figure S15 (Supporting Information). Figure $4 \mathrm{c}$ shows a schematic illustration of the flat-band energy level diagram of the layers. The poly-TPD layer, serving as both the hole-transporting and the electron-blocking layer, benefits from its specific molecular energy configuration. The highest occupied molecular orbital (HOMO, $5.4 \mathrm{eV}$ ) is located between the HOMO of PEDOT:PSS and the valance band of $\mathrm{CsPbBr}_{3}$, while the lowest unoccupied molecular orbital $(2.3 \mathrm{eV})$ is much higher than the $\mathrm{CsPbBr}$ conduction band. ${ }^{44}$ The TPBi layer worked as not only the electron transporting layer but also the hole blocking layer owing to its deep HOMO of $6.2 \mathrm{eV}^{45}$ Hence, holes and electrons were allowed to recombine effectively in the $\mathrm{Cs} \mathrm{PbBr}_{3}$-based emitting layer.

The performances of device G1 and device G2 are summarized in Figure 5 and Table 1. The maximum EQE for device G1 is as high as $1.70 \%$, which is about 34 -fold higher than that of device G2, as shown in Figure 5a. The remarkable EQE enhancement indicates that the carrier transport properties in the $\mathrm{Cs} \mathrm{PbBr}_{3}$-emitting layer were significantly improved because of the effect of the solvent-assisted surface engineering on the thin film forming. In addition, the EQE of $1.70 \%$ is also higher than those of recently reported representative $\mathrm{CsPbr}_{3}$ based LEDs (e.g., 1.49\% from Zeng's group, ${ }^{46} 1.37 \%$ from Lee's
Table 1. Summary of the Performances of LEDs

$\begin{array}{cccccc}\text { device } & \begin{array}{c}\mathrm{EQE}_{\max }^{a} \\ (\%)\end{array} & \begin{array}{c}\mathrm{CE}_{\max }{ }^{b} \\ \left(\mathrm{~cd} \mathrm{~A}^{-1}\right)\end{array} & \begin{array}{c}\mathrm{PE}_{\max }^{c} \\ \left(\mathrm{~lm} \mathrm{~W}^{-1}\right)\end{array} & \begin{array}{c}L_{\max }{ }^{d} \\ \left(\mathrm{~cd} \mathrm{~m}^{-2}\right)\end{array} & \begin{array}{c}\mathrm{fwhm} \\ (\mathrm{nm})\end{array} \\ \mathrm{G} 1 & 1.70 & 5.57 & 1.59 & 1562 & 16 \\ \mathrm{G} 2 & 0.05 & 0.18 & 0.04 & 140 & 16\end{array}$

${ }^{a}$ Maximum EQE. ${ }^{b}$ Maximum CE. ${ }^{c}$ Maximum PE. ${ }^{d}$ Maximum luminance.

group, ${ }^{47}$ and $\leq 0.93 \%$ for others $),{ }^{30,48-53}$ further indicating the advantage of the solvent-assisted surface engineering. It is worthy of noting that the EQE roll-off is quite obvious for both devices G1 and G2, which is a common phenomenon occurring in all-inorganic PeLEDs devices. ${ }^{46-53}$ There are several reasons that could lead to the roll-off case, including the current damage, the charging effect, the Auger decay, and the accumulation of mobile ion-induced luminescence quenching, which calls for further investigation. ${ }^{7,45,54}$ Both the current density and luminance intensify with the increase of the applied voltage, as presented in Figure $5 \mathrm{~b}$. The maximum luminance for device G1 is $1562 \mathrm{~cd} \mathrm{~m}^{-2}$, which is about 11-fold higher than that for device G2. The corresponding current density for device G1 has also been substantially improved, for instance, from $28 \mathrm{~mA} \mathrm{~cm}^{-2}$ for device $\mathrm{G} 2$ to $101 \mathrm{~mA} \mathrm{~cm}^{-2}$ at $14 \mathrm{~V}$. The maximum current efficiency for device G1 shows a maximum of $5.57 \mathrm{~cd} \mathrm{~A} \mathrm{~A}^{-1}$, which is 31 -fold higher than that of device G2 (Figure S16, Supporting Information). ${ }^{55-57}$

All these results confirm that the charge injection and transport balance have indeed been boosted after the process of solvent-assisted surface engineering. Figure $5 \mathrm{c}$ shows that the device gives a very narrow EL emission peak with a fwhm of 16 $\mathrm{nm}$ and it is, to the best of our knowledge, the narrowest value among $\mathrm{CsPbBr}$-based LEDs, which is attributed to the narrow band-edge emission of the $\mathrm{CsPbBr}_{3}$ nanocrystals. It's obvious that there is no parasitic emission originated from the chargetransport layers, indicating good electron and hole blocking functions of both poly-TPD and TPBi layers. It shows in Figure $5 \mathrm{~d}$ that the maximum power efficiency of device G1 is $1.59 \mathrm{~lm}$ 
$\mathrm{W}^{-1}$, which is 40 -fold higher than that of device $\mathrm{G} 2$ and further demonstrates the merit of the proposed strategy.

The operational stability of PeLEDs is a critical issue, which is rarely reported in this field. To deeply comprehend the asfabricated PeLEDs, the stability under continuous operation at a constant voltage of $10 \mathrm{~V}$ has been evaluated in ambient air at room temperature. The half-lifetime $\left(T_{50}\right)$ is defined as the time for the luminance decreasing to $50 \%$ of the starting luminance $\left(L_{0}\right)$. As shown in Figure S17 (Supporting Information), the $T_{50}$ of device G1 is $630 \mathrm{~s}$, which is better than previous allinorganic PeLEDs. ${ }^{58,59}$ On the other hand, the $T_{50}$ of device G2 is only $35 \mathrm{~s}$ at the same initial luminance, which is 18 -fold shorter than that of device G1, further indicating the advantage of self-assembled $\mathrm{CsPBr}_{3}$ thin films. The reproducibility of devices is reasonably high. It is demonstrated that the EQE values of over $80 \%$ of PeLED devices based on the selfassembled $\mathrm{CsPbBr}_{3}$ thin film were higher than $1.4 \%$, as shown by the histograms of maximum EQE taken from 18 devices (Figure S18, Supporting Information).

\section{CONCLUSIONS}

In summary, we have proposed and demonstrated a facile strategy, solvent-assisted surface engineering, to improve the quality of nanocrystal thin films for LED application. Solvents with different polarities were collaboratively utilized to obtain $\mathrm{Cs} \mathrm{PbBr}_{3}$ nanocrystal assemblies. The influence of the solventassisted surface engineering strategy on the morphology and optical and electronic properties of the nanocrystal thin films has been investigated. The solid $\mathrm{CsPbBr}_{3}$ thin films fabricated from the as-processed solution are highly dense and smooth, favorable for both charge injection and electrical transport for device application. Meanwhile, the thin films' photostability has been improved about 30\% compared to those made from pristine and unassembled $\mathrm{Cs} \mathrm{PbBr}_{3}$ nanoparticle solutions. Our strategy has been clearly validated by successful fabrication of $\mathrm{CsPbB}_{3}$ LEDs with remarkably high performance. Consequently, a 34-fold EQE enhancement (up to $1.70 \%$ ) has been achieved from solution-processed $\mathrm{CsPbBr}_{3}$ LEDs. The corresponding luminance, current efficiency, and the power efficiency were 11-, 31-, and 40-fold, respectively, which are much better than control devices. The fwhm of the EL spectrum is only $16 \mathrm{~nm}$, which is the narrowest among $\mathrm{CsPBr}_{3}$ LEDs. Our method is facile and reliable, thus the work shall be insightful for developing optoelectronic devices from the rising material system-inorganic metal lead halide perovskites.

\section{EXPERIMENTAL METHODS}

Chemicals. All reagents were used as received without further purification. 1-Octadecene (ODE, technical grade, 90\%), OA (technical grade, 90\%), OLA (technical grade, 70\%), cesium carbonate $\left(\mathrm{Cs}_{2} \mathrm{CO}_{3}, 99.9 \%\right.$ trace metals basis), lead chloride $\left(\mathrm{PbCl}_{2}, 99.999 \%\right.$ trace metals basis), lead bromide ( $\mathrm{PbBr}_{2}, 99.999 \%$ trace metals basis), lead iodide $\left(\mathrm{PbI}_{2}, 99.999 \%\right.$ trace metals basis), EA (anhydrous, 99.8\%), toluene (anhydrous), and octane (anhydrous, $\geq 99 \%$ ) were all purchased from Sigma-Aldrich. Hexane (HPLC grade) was purchased from Fishier Scientific.

Synthesis of Cs-Oleate. $\mathrm{Cs}_{2} \mathrm{CO}_{3}(0.814 \mathrm{~g}), 40 \mathrm{~mL}$ ODE, and 2.5 $\mathrm{mL}$ OA were loaded into a $100 \mathrm{~mL}$ three-neck flask, dried at $120{ }^{\circ} \mathrm{C}$ for $1 \mathrm{~h}$, and then the temperature was raised to $150{ }^{\circ} \mathrm{C}$ under $\mathrm{N}_{2}$ flow until all $\mathrm{Cs}_{2} \mathrm{CO}_{3}$ reacted with OA. It needs to be preheated to $150{ }^{\circ} \mathrm{C}$ before injection.

Synthesis of $\mathrm{CsPbBr}_{3}$ QDs. ODE (15 mL), $3 \mathrm{~mL}$ of OAm, $1.5 \mathrm{~mL}$ of $\mathrm{OA}$, and $\mathrm{PbBr}_{2}(0.2 \mathrm{~g})$ were loaded into a $50 \mathrm{~mL}$ three-neck flask, degassed, and dried under vacuum for $20 \mathrm{~min}$ at $120{ }^{\circ} \mathrm{C}$. During this process, the $\mathrm{PbBr}_{2}$ precursor dissolved completely and mixed thoroughly with the reaction solution. Then, with the protection of $\mathrm{N}_{2}$ atmosphere, the reaction temperature was raised to $170{ }^{\circ} \mathrm{C}$ with a rate of $15^{\circ} \mathrm{C} \mathrm{min}^{-1}$. The preheated Cs-oleate solution $(0.66 \mathrm{~mL}, 0.125$ $\mathrm{M}$ in ODE) was injected swiftly, $8 \mathrm{~s}$ later, and the reaction mixture was cooled down with a rate of $15{ }^{\circ} \mathrm{C} \mathrm{min}{ }^{-1}$ to the room temperature.

Processes for Partial Connected of $\mathrm{CsPbBr}_{3}$ QDs. The purification process contains mainly three steps: hexane was first added into the crude solution with a volume ratio of $(1: 10)$, and the precipitate was collected after centrifugation $(8500 \mathrm{rpm}, 5 \mathrm{~min})$ and dispersed in $4 \mathrm{~mL}$ hexane. Then, $16 \mathrm{~mL}$ of EA was added into $4 \mathrm{~mL}$ of hexane solution with a volume ratio of $4: 1$ and the precipitate was collected after centrifugation $(8500 \mathrm{rpm}, 5 \mathrm{~min})$ and redispersed in 4 $\mathrm{mL}$ of hexane. The final product was saved as stock solution for the LED fabrication.

Optical Characterization. UV-vis absorption spectra were collected with a UV-1800 spectrometer. The PLQYs of solution samples were obtained at an excitation wavelength of $442 \mathrm{~nm}$ with the absolute quantum efficiency measurement system-QE 200B (Otsuka Electronic Co., Ltd.) To characterize the photostability of the thin film sample, the samples were fixed and irradiated by the $\mathrm{He}-\mathrm{Cd}$ continuous wave laser light $(442 \mathrm{~nm})$ for $90 \mathrm{~min}$. The PL signal was dispersed by a monochromator and detected by a photomultiplier tube.

Device Fabrication and Characterization. PEDOT:PSS solutions (filtered through a $0.45 \mu \mathrm{m}$ filter) were spin-coated onto the ITO-coated glass substrates (having a sheet resistance of $15 \Omega$ $\mathrm{sq}^{-1}$ ) at $4000 \mathrm{rpm}$ for $60 \mathrm{~s}$ and baked at $150{ }^{\circ} \mathrm{C}$ for $30 \mathrm{~min}$. The hole transporting and electron blocking layers were prepared by spincoating poly-TPD chlorobenzene solution (concentration: $8 \mathrm{mg}$ $\mathrm{mL}^{-1}$ ) at $2000 \mathrm{rpm}$ for $45 \mathrm{~s}$. Perovskite nanocrystals were deposited by spin-coating at $2000 \mathrm{rpm}$ for $45 \mathrm{~s}$. Afterward, the samples were transferred into a vacuum thermal evaporation chamber to deposit other organic materials and cathode. Without breaking the vacuum, the $\mathrm{TPBi}, \mathrm{Cs}_{2} \mathrm{CO}_{3}$, and $\mathrm{Al}$ layers were thermally deposited at a base pressure of $4.0 \times 10^{-4} \mathrm{~Pa}$. The thicknesses of layers were controlled by the quartz crystal oscillators. After preparation under a nitrogen atmosphere using epoxy glue and glass slides, all devices were encapsulated immediately. The emission area of all devices is $1 \times 1$ $\mathrm{mm}^{2}$ as defined by the overlapping area of the anode and the cathode. The EL spectra were recorded via a PR705 Spectra Scan spectrometer. By utilizing a computer-controlled source meter (Keithley source measure unit 2400), the current density-voltage-luminance $(I-V-L)$ properties were measured simultaneously. Accordingly, the CE and PE were obtained. EQE values were calculated from the luminance, current density, and EL spectrum.

\section{ASSOCIATED CONTENT}

Supporting Information

The Supporting Information is available free of charge on the ACS Publications website at DOI: 10.1021/acsami.8b06105.

$$
\text { Additional figures (PDF) }
$$

\section{AUTHOR INFORMATION}

\section{Corresponding Authors}

*E-mail: hvdemir@ntu.edu.sg (H.V.D.).

*E-mail: guhsh@hubu.edu.cn (H.G.).

*E-mail: hdsun@ntu.edu (H.S.).

ORCID $\odot$

Hilmi Volkan Demir: 0000-0003-1793-112X

Haoshuang Gu: 0000-0003-1232-2499

Handong Sun: 0000-0002-2261-7103

\section{Author Contributions}

${ }^{\mathrm{II}}$ L.W. and B.L. make contribution equally.

Notes

The authors declare no competing financial interest. 


\section{ACKNOWLEDGMENTS}

This work was supported by the Singapore Ministry of Education through the Academic Research Fund under Projects MOE2016-T2-1-054, Tier 1-RG105/16 and Tier 1-RG92/15 and China Scholarship Council (20163100, no. 201608420137). Thanks to the support of the National Research Foundation, Prime Minister's Office, Singapore under its Competitive Research program (NRF-CRP14-201403), its Investigatorship program (NRF-NRFI2016-08) and the Singapore Agency for Science, Technology and Research (A*STAR) SERC Pharos Program under grant no. 15273 00025. H.V.D. gratefully acknowledges TUBA-GEBIP.

\section{REFERENCES}

(1) Protesescu, L.; Yakunin, S.; Bodnarchuk, M. I.; Krieg, F.; Caputo, R.; Hendon, C. H.; Yang, R. X.; Walsh, A.; Kovalenko, M. V. Nanocrystals of Cesium Lead Halide Perovskites $\left(\mathrm{CsPbX}_{3}, \mathrm{X}=\mathrm{Cl}, \mathrm{Br}\right.$, and I): Novel Optoelectronic Materials Showing Bright Emission with Wide Color Gamut. Nano Lett. 2015, 15, 3692-3696.

(2) Ramasamy, P.; Lim, D.-H.; Kim, B.; Lee, S.-H.; Lee, M.-S.; Lee, J.S. All-inorganic Cesium Lead Halide Perovskite Nanocrystals for Photodetector Applications. Chem. Commun. 2015, 52, 2067-2070.

(3) Li, X.; Wu, Y.; Zhang, S.; Cai, B.; Gu, Y.; Song, J.; Zeng, H. $\mathrm{CsPbX}_{3}$ Quantum Dots for Lighting and Displays: Room-Temperature Synthesis, Photoluminescence Superiorities, Underlying Origins and White Light-Emitting Diodes. Adv. Funct. Mater. 2016, 26, 24352445.

(4) Parobek, D.; Dong, Y.; Qiao, T.; Rossi, D.; Son, D. H. Photoinduced Anion Exchange in Cesium Lead Halide Perovskite Nanocrystals. J. Am. Chem. Soc. 2017, 139, 4358-4361.

(5) Rakita, Y.; Kedem, N.; Gupta, S.; Sadhanala, A.; Kalchenko, V.; Böhm, M. L.; Kulbak, M.; Friend, R. H.; Cahen, D.; Hodes, G. LowTemperature Solution-Grown $\mathrm{CsPbBr}_{3}$ Single Crystals and Their Characterization. Cryst. Growth Des. 2016, 16, 5717-5725.

(6) Nedelcu, G.; Protesescu, L.; Yakunin, S.; Bodnarchuk, M. I.; Grotevent, M. J.; Kovalenko, M. V. Fast Anion-Exchange in Highly Luminescent Nanocrystals of Cesium Lead Halide Perovskites $\left(\mathrm{CsPbX}_{3}, \mathrm{X}=\mathrm{Cl}, \mathrm{Br}, \mathrm{I}\right)$. Nano Lett. 2015, 15, 5635-5640.

(7) Song, J.; Li, J.; Li, X.; Xu, L.; Dong, Y.; Zeng, H. Quantum Dot Light-Emitting Diodes Based on Inorganic Perovskite Cesium Lead Halides $\left(\mathrm{CsPbX}_{3}\right)$. Adv. Mater. 2015, 27, 7162-7167.

(8) Berends, A. C.; de Mello Donega, C. Ultrathin One- and TwoDimensional Colloidal Semiconductor Nanocrystals: Pushing Quantum Confinement to the Limit. J. Phys. Chem. Lett. 2017, 8, 40774090.

(9) Zhang, D.; Eaton, S. W.; Yu, Y.; Dou, L.; Yang, P. Solution-Phase Synthesis of Cesium Lead Halide Perovskite Nanowires. J. Am. Chem. Soc. 2015, 137, 9230-9233.

(10) Wang, Y.; Li, X.; Sreejith, S.; Cao, F.; Wang, Z.; Stuparu, M. C.; Zeng, H.; Sun, H. Photon Driven Transformation of Cesium Lead Halide Perovskites from Few-Monolayer Nanoplatelets to Bulk Phase. Adv. Mater. 2016, 28, 10637-10643.

(11) Akkerman, Q. A.; Motti, S. G.; Kandada, A. R. S.; Mosconi, E.; D’Innocenzo, V.; Bertoni, G.; Marras, S.; Kamino, B. A.; Miranda, L.; De Angelis, F.; Petrozza, A.; Prato, M.; Manna, L. Solution Synthesis Approach to Colloidal Cesium Lead Halide Perovskite Nanoplatelets with Monolayer-Level Thickness Control by a Solution Synthesis Approach. J. Am. Chem. Soc. 2016, 138, 1010-1016.

(12) Wang, Y.; Sun, H. All-Inorganic Metal Halide Perovskite Nanostructures: From Photophysics to Light-Emitting Applications. Small Methods 2017, 2, 1700252.

(13) Zhang, X.; Sun, C.; Zhang, Y.; Wu, H.; Ji, C.; Chuai, Y.; Wang, P.; Wen, S.; Zhang, C.; Yu, W. W. Bright Perovskite Nanocrystal Films for Efficient Light-Emitting Devices. J. Phys. Chem. Lett. 2016, 7, $4602-4610$.

(14) Zhang, X.; Lin, H.; Huang, H.; Reckmeier, C.; Zhang, Y.; Choy, W. C. H.; Rogach, A. L. Enhancing the Brightness of Cesium Lead
Halide Perovskite Nanocrystal Based Green Light-Emitting Devices through the Interface Engineering with Perfluorinated Ionomer. Nano Lett. 2016, 16, 1415-1420.

(15) Zhang, L.; Yang, X.; Jiang, Q.; Wang, P.; Yin, Z.; Zhang, X.; Tan, H.; Yang, Y.; Wei, M.; Sutherland, B. R.; Sargent, E. H.; You, J. Ultrabright and Highly Efficient Inorganic Based Perovskite Light-emitting Diodes. Nat. Commun. 2017, 8, 15640.

(16) Kim, Y.-H.; Wolf, C.; Kim, Y.-T.; Cho, H.; Kwon, W.; Do, S.; Sadhanala, A.; Park, C. G.; Rhee, S.-W.; Im, S. H.; Friend, R. H.; Lee, T.-W. Highly Efficient Light-Emitting Diodes of Colloidal MetalHalide Perovskite Nanocrystals beyond Quantum Size. ACS Nano 2017, 11, 6586-6593.

(17) Yang, B.; Zhang, F.; Chen, J.; Yang, S.; Xia, X.; Pullerits, T.; Deng, W.; Han, K. Ultrasensitive and Fast All-Inorganic PerovskiteBased Photodetector via Fast Carrier Diffusion. Adv. Mater. 2017, 29, 1703758.

(18) Wang, H.; Kim, D. H. Perovskite-based Photodetectors: Materials and Devices. Chem. Soc. Rev. 2017, 46, 5204-5236.

(19) Akkerman, Q. A.; Gandini, M.; Di Stasio, F.; Rastogi, P.; Palazon, F.; Bertoni, G.; Ball, J. M.; Prato, M.; Petrozza, A.; Manna, L. Strongly Emissive Perovskite Nanocrystal Inks for High-Voltage Solar Cells. Nat. Energy 2016, 2, 16194.

(20) Zhang, X.; Zhang, J.; Phuyal, D.; Du, J.; Tian, L.; Öberg, V. A.; Johansson, M. B.; Cappel, U. B.; Karis, O.; Liu, J.; Rensmo, H.; Boschloo, G.; Johansson, E. M. J. Inorganic $\mathrm{CsPbI}_{3}$ Perovskite Coating on $\mathrm{PbS}$ Quantum Dot for Highly Efficient and Stable Infrared Light Converting Solar Cells. Adv. Energy Mater. 2017, 8, 1702049.

(21) Yakunin, S.; Protesescu, L.; Krieg, F.; Bodnarchuk, M. I.; Nedelcu, G.; Humer, M.; De Luca, G.; Fiebig, M.; Heiss, W.; Kovalenko, M. V. Low-threshold Amplified Spontaneous Emission and Lasing from Colloidal Nanocrystals of Caesium Lead Halide Perovskites. Nat. Commun. 2015, 6, 8056.

(22) Wang, Y.; Li, X.; Nalla, V.; Zeng, H.; Sun, H. Solution-Processed Low Threshold Vertical Cavity Surface Emitting Lasers from AllInorganic Perovskite Nanocrystals. Adv. Funct. Mater. 2017, 27, 1605088 .

(23) Wang, Y.; Li, X.; Song, J.; Xiao, L.; Zeng, H.; Sun, H. AllInorganic Colloidal Perovskite Quantum Dots: A New Class of Lasing Materials with Favorable Characteristics. Adv. Mater. 2015, 27, 71017108.

(24) Boles, M. A.; Ling, D.; Hyeon, T.; Talapin, D. V. The Surface Science of Nanocrystals. Nat. Mater. 2016, 15, 141-153.

(25) Cho, H.; Jeong, S.-H.; Park, M.-H.; Kim, Y.-H.; Wolf, C.; Lee, C.-L.; Heo, J. H.; Sadhanala, A.; Myoung, N.; Yoo, S.; Im, S. H.; Friend, R. H.; Lee, T.-W. Overcoming the Electroluminescence Efficiency Limitations of Perovskite Light-emitting Diodes. Science 2015, 350, 1222-1225.

(26) De Roo, J.; Ibáñez, M.; Geiregat, P.; Nedelcu, G.; Walravens, W.; Maes, J.; Martins, J. C.; Van Driessche, I.; Kovalenko, M. V.; Hens, Z. Highly Dynamic Ligand Binding and Light Absorption Coefficient of Cesium Lead Bromide Perovskite Nanocrystals. ACS Nano 2016, 10, 2071-2081.

(27) Kang, J.; Wang, L.-W. High Defect Tolerance in Lead Halide Perovskite CsPbBr. J. Phys. Chem. Lett. 2017, 8, 489-493.

(28) ten Brinck, S.; Infante, I. Surface Termination, Morphology, and Bright Photoluminescence of Cesium Lead Halide Perovskite Nanocrystals. ACS Energy Lett. 2016, 1, 1266-1272.

(29) Li, X.; Yu, D.; Cao, F.; Gu, Y.; Wei, Y.; Wu, Y.; Song, J.; Zeng, H. Healing All-Inorganic Perovskite Films via Recyclable DissolutionRecyrstallization for Compact and Smooth Carrier Channels of Optoelectronic Devices with High Stability. Adv. Funct. Mater. 2016, 26, 5903-5912.

(30) Zhang, X.; Wang, W.; Xu, B.; Liu, S.; Dai, H.; Bian, D.; Chen, S.; Wang, K.; Sun, X. W. Thin Film Perovskite Light-emitting Diode Based on $\mathrm{CsPbBr}_{3}$ Powders and Interfacial Engineering. Nano Energy 2017, 37, 40-45.

(31) Zhao, L.; Yeh, Y.-W.; Tran, N. L.; Wu, F.; Xiao, Z.; Kerner, R. A.; Lin, Y. L.; Scholes, G. D.; Yao, N.; Rand, B. P. In Situ Preparation 
of Metal Halide Perovskite Nanocrystal Thin Films for Improved Light-Emitting Devices. ACS Nano 2017, 11, 3957-3964.

(32) Yu, J. C.; Kim, D. W.; Kim, D. B.; Jung, E. D.; Lee, K.-S.; Lee, S.; Nuzzo, D. D.; Kim, J.-S.; Song, M. H. Effect of the Solvent Used for Fabrication of Perovskite Films by Solvent Dropping on Performance of Perovskite Light-emitting Diodes. Nanoscale 2017, 9, 2088-2094.

(33) Chiba, T.; Hoshi, K.; Pu, Y.-J.; Takeda, Y.; Hayashi, Y.; Ohisa, S.; Kawata, S.; Kido, J. High-Efficiency Perovskite Quantum-Dot Light-Emitting Devices by Effective Washing Process and Interfacial Energy Level Alignment. ACS Appl. Mater. Interfaces 2017, 9, 1805418060 .

(34) Pan, J.; Quan, L. N.; Zhao, Y.; Peng, W.; Murali, B.; Sarmah, S. P.; Yuan, M.; Sinatra, L.; Alyami, N. M.; Liu, J.; Yassitepe, E.; Yang, Z.; Voznyy, O.; Comin, R.; Hedhili, M. N.; Mohammed, O. F.; Lu, Z. H.; Kim, D. H.; Sargent, E. H.; Bakr, O. M. Highly Efficient PerovskiteQuantum-Dot Light-Emitting Diodes by Surface Engineering. Adv. Mater. 2016, 28, 8718-8725.

(35) Soetan, N.; Erwin, W. R.; Tonigan, A. M.; Walker, D. G.; Bardhan, R. Solvent-Assisted Self-Assembly of $\mathrm{CsPbBr}_{3}$ Perovskite Nanocrystals into One-Dimensional Superlattice. J. Phys. Chem. C 2017, 121, 18186-18194.

(36) Li, J.; Xu, L.; Wang, T.; Song, J.; Chen, J.; Xue, J.; Dong, Y.; Cai, B.; Shan, Q.; Han, B.; Zeng, H. 50-Fold EQE Improvement up to $6.27 \%$ of Solution-Processed All-Inorganic Perovskite CsPbBr 3 QLEDs via Surface Ligand Density Control. Adv. Mater. 2017, 29, 1603885.

(37) Boles, M. A.; Engel, M.; Talapin, D. V. Self-Assembly of Colloidal Nanocrystals: From Intricate Structures to Functional Materials. Chem. Rev. 2016, 116, 11220-11289.

(38) Cademartiri, L.; Bishop, K. J. M.; Snyder, P. W.; Ozin, G. A. Using Shape for Self-assembly. Philos. Trans. R. Soc., A 2012, 370, 2824-2847.

(39) Ravi, V. K.; Santra, P. K.; Joshi, N.; Chugh, J.; Singh, S. K.; Rensmo, H.; Ghosh, P.; Nag, A. Origin of the Substitution Mechanism for the Binding of Organic Ligands on the Surface of $\mathrm{CsPBr}_{3}$ Perovskite Nanocubes. J. Phys. Chem. Lett. 2017, 8, 4988-4994.

(40) Sperling, R. A.; Parak, W. J. Surface Modification, Functionalization and Bioconjugation of Colloidal Inorganic Nanoparticles. Philos. Trans. R. Soc., A 2010, 368, 1333-1383.

(41) Pradhan, N.; Reifsnyder, D.; Xie, R.; Aldana, J.; Peng, X. Surface Ligand Dynamics in Growth of Nanocrystals. J. Am. Chem. Soc. 2007, 129, 9500-9509.

(42) Swarnkar, A.; Marshall, A. R.; Sanehira, E. M.; Chernomordik, B. D.; Moore, D. T.; Christians, J. A.; Chakrabarti, T.; Luther, J. M. Quantum Dot-Induced Phase Stabilization of a-CsPbI Perovskite for High-efficiency Photovoltaics. Science 2016, 354, 92-95.

(43) Chen, J.; Liu, D.; Al-Marri, M. J.; Nuuttila, L.; Lehtivuori, H.; Zheng, K. Photo-stability of $\mathrm{CsPbBr}_{3}$ Perovskite Quantum Dots for Optoelectronic Application. Sci. China Mater. 2016, 59, 719-727.

(44) Wang, M.; Hill, I. G. Fluorinated Alkyl Phosphonic Acid SAMs Replace PEDOT: PSS in Polymer Semiconductor Devices. Org. Electron. 2012, 13, 498-505.

(45) Liu, B.; Nie, H.; Zhou, X.; Hu, S.; Luo, D.; Gao, D.; Zou, J.; Xu, M.; Wang, L.; Zhao, Z.; Qin, A.; Peng, J.; Ning, H.; Cao, Y.; Tang, B. Z. Manipulation of Charge and Exciton Distribution Based on Blue Aggregation-Induced Emission Fluorophors: A Novel Concept to Achieve High-Performance Hybrid White Organic Light-Emitting Diodes. Adv. Funct. Mater. 2016, 26, 776-783.

(46) Zou, S.; Liu, Y.; Li, J.; Liu, C.; Feng, R.; Jiang, F.; Li, Y.; Song, J.; Zeng, H.; Hong, M.; Chen, X. Stabilizing Cesium Lead Halide Perovskite Lattice through Mn (II) Substitution for Air-Stable LightEmitting Diodes. J. Am. Chem. Soc. 2017, 139, 11443-11450.

(47) Cho, H.; Wolf, C.; Kim, J. S.; Yun, H. J.; Bae, J. S.; Kim, H.; Heo, J.-M.; Ahn, S.; Lee, T.-W. High-Efficiency Solution-Processed Inorganic Metal Halide Perovskite Light-Emitting Diodes. Adv. Mater. 2017, 29, 1700579.

(48) Van Le, Q.; Kim, J. B.; Kim, S. Y.; Lee, B.; Lee, D. R. Structural Investigation of Cesium Lead Halide Perovskites for High-Efficiency Quantum Dot Light-Emitting Diodes. J. Phys. Chem. Lett. 2017, 8, 4140-4147.
(49) Zhang, X.; Xu, B.; Wang, W.; Liu, S.; Zheng, Y.; Chen, S.; Wang, K.; Sun, X. W. Plasmonic Perovskite Light-Emitting Diodes Based on the Ag-CsPbBr 3 System. ACS Appl. Mater. Interfaces 2017, 9, 49264931.

(50) Shan, Q.; Li, J.; Song, J.; Zou, Y.; Xu, L.; Xue, J.; Dong, Y.; Huo, C.; Chen, J.; Han, B.; Zeng, H. All-inorganic Quantum-dot Lightemitting Diodes Based on Perovskite Emitters with Low Turn-on Voltage and High Humidity Stability. J. Mater. Chem. C 2017, 5, $4565-4570$

(51) Wei, S.; Yang, Y.; Kang, X.; Wang, L.; Huang, L.; Pan, D. Homogeneous Synthesis and Electroluminescence Device of Highly Luminescent $\mathrm{CsPbBr} 3$ Perovskite Nanocrystals. Inorg. Chem. 2017, 56, 2596-2601.

(52) Van Le, Q.; Park, M.; Sohn, W.; Jang, H. W.; Kim, S. Y. Investigation of Energy Levels and Crystal Structures of Cesium Lead Halides and Their Application in Full-Color Light-Emitting Diodes. Adv. Electron. Mater. 2017, 3, 1600448.

(53) Du, X.; Wu, G.; Cheng, J.; Dang, H.; Ma, K.; Zhang, Y.-W.; Tan, P.-F.; Chen, S. High-Quality $\mathrm{CsPbBr}_{3}$ Perovskite Nanocrystals for Quantum Dot Light-emitting Diodes. RSC Adv. 2017, 7, 1039110396.

(54) Liu, B.; Wang, L.; Gao, D.; Xu, M.; Zhu, X.; Zou, J.; Lan, L.; Ning, H.; Peng, J.; Cao, Y. Harnessing charge and exciton distribution towards extremely high performance: the critical role of guests in single-emitting-layer white OLEDs. Mater. Horiz. 2015, 2, 536-544.

(55) Shi, Z.; Li, Y.; Zhang, Y.; Chen, Y.; Li, X.; Wu, D.; Xu, T.; Shan, C.; Du, G. High-efficiency and air-stable perovskite quantum dots light-emitting diodes with an all-inorganic heterostructure. Nano Lett. 2017, 17, 313-321.

(56) Shi, Z.; Li, S.; Li, Y.; Ji, H.; Li, X.; Wu, D.; Xu, T.; Chen, Y.; Tian, Y.; Zhang, Y.; Shan, C.; Du, G. A Strategy of Solution-Processed All-Inorganic-Heterostructure for Humidity/Temperature-Stable Perovskite Quantum Dot Light-Emitting Diodes. ACS Nano 2018, 12, $1462-1472$.

(57) Shi, Z.; Li, Y.; Li, S.; Li, X.; Wu, D.; Xu, T.; Tian, Y.; Chen, Y.; Zhang, Y.; Zhang, B.; Shan, C.; Du, G. Localized Surface Plasmon Enhanced All-Inorganic Perovskite Quantum Dot Light-Emitting Diodes Based on Coaxial Core/Shell Heterojunction Architecture. Adv. Funct. Mater. 2018, 28, 1707031.

(58) Zhang, X.; Lin, H.; Huang, H.; Reckmeier, C.; Zhang, Y.; Choy, W. C. H.; Rogach, A. L. Enhancing the brightness of cesium lead halide perovskite nanocrystal based green light-emitting devices through the interface engineering with perfluorinated ionomer. Nano Lett. 2016, 2, 1415-1420.

(59) Liu, B.; Wang, L.; Gu, H.; Sun, H.; Demir, H. V. Highly Efficient Green Light-Emitting Diodes from All-Inorganic Perovskite Nanocrystals Enabled by a New Electron Transport Layer. Adv. Opt. Mater. 2018, 1800220. 